\title{
Sunquakes and starquakes
}

\author{
Alexander G. Kosovichev ${ }^{1,2,3}$ \\ ${ }^{1}$ Hansen Experimental Physics Laboratory, Stanford University, Stanford, CA 94305, USA \\ email: AKosovichev@solar.stanford.edu \\ ${ }^{2}$ Big Bear Solar Observatory, Big Bear City, CA 92314, USA \\ ${ }^{3}$ Crimean Astrophysical Observatory, Nauchny, Crimea 98409, Ukraine
}

\begin{abstract}
In addition to well-known mechanisms of excitation of solar and stellar oscillations by turbulent convection and instabilities, the oscillations can be excited by an impulsive localized force caused by the energy release in solar and stellar flares. Such oscillations have been observed on the Sun ('sunquakes'), and created a lot of interesting discussions about physical mechanisms of the impulsive excitation and their relationship to the flare physics. The observation and theory have shown that most of a sunquake's energy is released in high-degree, high-frequency p modes. In addition, there have been reports on helioseismic observations of low-degree modes excited by strong solar flares. Much more powerful flares observed on other stars can cause 'starquakes' of substantially higher amplitude. Observations of such oscillations can provide new asteroseismic information and also constraints on mechanisms of stellar flares. I discuss the basic properties of sunquakes, and initial attempts to detect flare-excited oscillations in Kepler short-cadence data.
\end{abstract}

Keywords. Sun: flares, oscillations; stars: flares, oscillations

\section{Introduction}

Kepler observations have led to a discovery that stellar flares representing impulsive powerful energy releases occur not only in M-type dwarfs (UV Ceti-type variables) but also in a wide range of A-F type stars (Balona 2012; Maehara et al. 2012; Walkowicz et al. 2011). Previously, it was believed the F- and A-type stars do not have flaring activity. The discovery of super-flares on solar-type stars raised questions about the possibility of such flares on the Sun. These results triggered new debates about the physical mechanism of stellar flares and the relationship to the dynamo mechanism. Recent observations of solar flares from the Solar Dynamics Observatory (SDO) have found that the white-light flares are often accompanied by excitation of acoustic oscillations ( $\mathrm{p}$ modes) in the solar interior, so-called 'sunquakes'. However, not all flares reveal the sunquakes. The relationship of the interior and atmospheric response to the energy release and optical emission is not understood, and is currently a subject of detailed studies in heliophysics. The flare-excited oscillations are mostly observed as local seismic waves, and while the theory predicts that the global acoustic waves are also excited, their amplitude is significantly lower than the amplitude of stochastically excited oscillations, therefore, the detection reports have been controversial. However, in the case of significantly more powerful stellar flares the impact on the star's surface is much greater. This can lead to excitation of the global low-degree oscillations to significantly higher amplitudes than on the Sun. Our preliminary study of the available short-cadence (SC) data provides indications of such 'starquakes'. However, a statistical study for a large sample of stars and longer observing intervals are needed. 

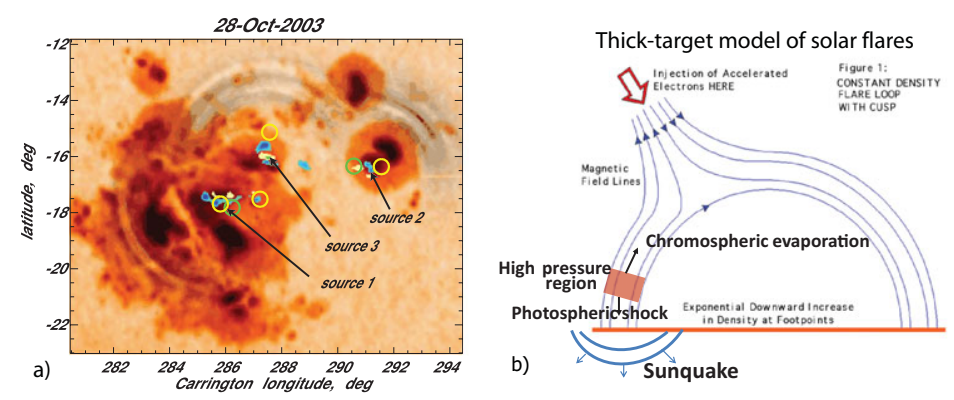

Figure 1. a) Observations of acoustic wavefronts (projected onto a sunspot image) during a large solar flare. Yellow and green circles show places of the hard X-ray and $\gamma$-ray emissions, and blue patches are Doppler-shift signals $>1 \mathrm{~km} \mathrm{~s}^{-1}$ from the localized flare impacts (Kosovichev $2006)$; b) illustration of excitation of 'sunquakes' in the 'thick-target' model of solar flares.

\section{Solar flares and sunquakes}

Recent observations of solar flares on the NASA space missions $S O H O$ and SDO revealed that some flares result in excitation of acoustic oscillations in the solar interior, dubbed 'sunquakes'. The oscillations are observed in the Doppler shift and intensity variations as expanding circular waves (Fig. 1a). They are excited due to momentum and energy impulse in the solar photosphere, which is also observed in solar flares, but the mechanism of this impact is unknown. The expanding waves represent high-degree $p$ modes; however, the stellar oscillation theory predicts that the global low-degree modes are also excited. Their detection has been reported from a statistical analysis of the correlation between the flare soft X-ray signals (observed by the GOES satellites) and the total solar irradiance measurements from the space observatory $S O H O$ (Karoff \& Kjeldsen 2008). However, there was no unambiguous detection of the whole-Sun oscillations caused by individual flare events. Indeed, the oscillation theory predicts that the amplitudes of the low-degree modes of sunquakes are significantly lower than the amplitudes of stochastically excited oscillations (Fig. 2a) (Kosovichev 2009).

The solar observations show that the sunquake events are mostly associated with compact or confined flares that do not produce coronal mass ejections and are characterized by the energy release in compact magnetic configurations in the lower atmosphere. Such flares usually produce white-light or continuum optical emission, and they do not necessarily have strong X-ray emission. The origin of such flare energy release is mysterious, and the subject of intensive investigation. These flares and sunquakes challenge the standard 'thick-target' flare model, which assumes that most of the flare energy is released in the form of high-energy particles due to magnetic reconnection processes in the coronal plasma. The particles travel along the magnetic field lines and heat the upper chromosphere to high temperature, creating a high-pressure region, which expands, producing a plasma eruption ('chromospheric evaporation') and a downward-traveling radiative shock (Fig. 1b). This shock can reach the photosphere and excite acoustic oscillations. However, the numerical simulations of the flare hydrodynamics show that the energy of the shock may be not sufficient to explain the observed oscillations, and also the photospheric impact is often observed at the beginning of the flare impulsive phase or even during the pre-heating phase, well before the maximum of the hard and soft X-ray emissions that are supposedly produced by the particle interaction with the higher chromosphere. Nevertheless, if a similar energy release mechanism works on other stars then the amplitudes of the flare-excited oscillations can be expected to be several orders of magnitude stronger than on the Sun. 

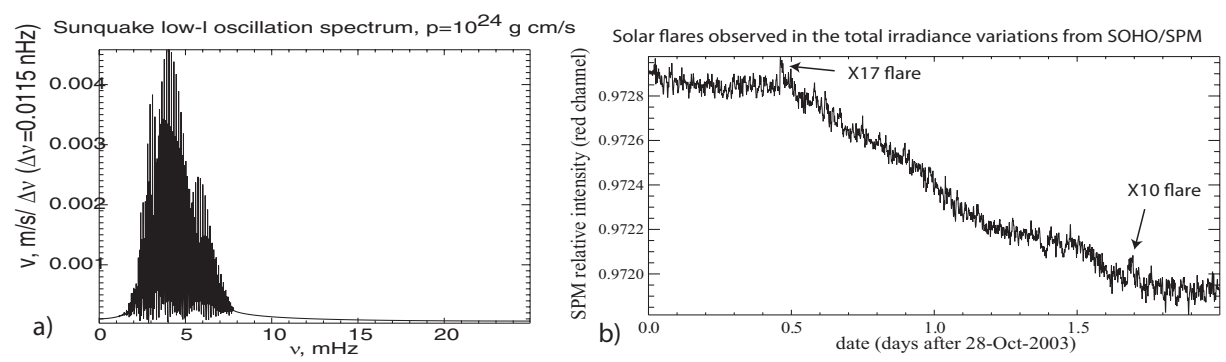

Figure 2. a) The theoretical spectrum of the flare excited oscillations has a maximum around the acoustic cut-off frequency $\sim 5 \mathrm{mHz}$ (Kosovichev 2009); (b) The signal of two large solar flares observed in the total irradiance measurements (red channel) with the SPM instrument on the SOHO spacecraft.

\section{Initial analysis of starquakes}

Observations of stellar flares from Kepler provide a unique opportunity to investigate the relationship between the flare energy release and oscillations. The analysis of Kepler data by Balona (2012), Maehara et al. (2012) and Walkowicz et al. (2011) has led to the discovery of a large number of flaring stars of various spectral classes, from M- to A-class. While large flares on M-K stars were known from ground-based observations, the discovery of similar flares on F-A stars is surprising. However, most of the flare data were obtained with long cadence, and do not provide sufficient resolution to estimate the flare amplitude and duration of the various phases of the energy release, or to investigate the seismic response of the stars. For understanding the mechanisms of flares and the impact of these flares, it is important to obtain more short-cadence data for flaring stars.

The previous Kepler observations allowed us to select stars that showed multiple flares and with good quality oscillation data (when short-cadence runs are available). We visually inspected the light curves of the previously detected flaring stars, as well as the database of the ground observations.

The flare brightness signals observed by Kepler (as illustrated in Fig. 3a) are much stronger than those on the Sun (Fig. 2a). Thus, the photospheric impacts can be much stronger, and the flare oscillations can be observed in the Kepler data. In Fig. 3 we illustrate the potential detection of a 'starquake' event obtained from the SC Kepler observations of KIC 6106415. The Kepler light curve of this stellar flare is shown in panel (a). The oscillation power spectrum calculated for a 2-day period immediately after the flare (Fig. 3b) shows an enhancement of the p-mode amplitudes at $\sim 2.6 \mathrm{mHz}$, closer to the acoustic cut-off frequency for this star $(\sim 3.5 \mathrm{mHz})$, compared to the oscillation spectrum taken during the star's quiet period (Fig. 3c), more than 5 days after the flare (the so-called 'numax' parameter at a frequency of $\sim 2.26 \mathrm{mHz}$, e.g., see Chaplin et al., 2014). Of course, more statistical studies are needed to confirm this result.

Oscillations associated with stellar flares have previously been observed in optical and X-ray emissions with periods ranging from a few seconds to tens of minutes, and have usually been interpreted as transient oscillations of flaring loops in stellar atmospheres (Contadakis et al. 2012; Jakimiec \& Tomczak 2012; Koljonen et al. 2011; Contadakis 2012; Qian et al. 2012). However, the atmospheric transient oscillations quickly decay, within 1-2 hours after a flare impulse (Bryson et al. 2005), while the stellar oscillation modes will live for much longer, depending on the damping mechanism in stellar envelopes. Also, the maximum amplitude of the oscillations modes excited by flares is expected to be in the range of $3-20 \mathrm{~min}$, close to the cut-off period of acoustic oscillations. We cannot rule out that oscillations with longer periods, corresponding to g modes and mixed modes, 

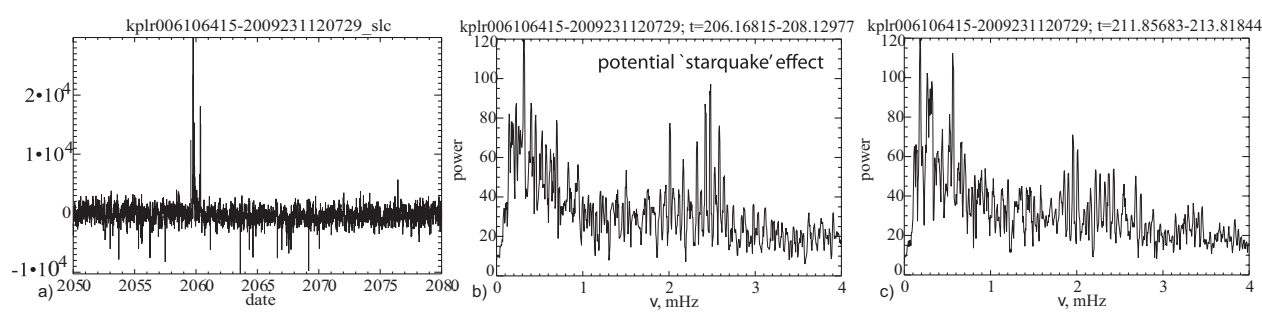

Figure 3. Illustration of a 'starquake' candidate obtained from the SC Kepler observations of KIC 6106415: (a) the Kepler light curve of a stellar flare. The oscillation power spectra: (b) calculated for a 2-day period immediately after the flare, and (c) taken during the star quiet period, more than 5 days after the flare.

are also excited. Nevertheless, we plan the initial search for the flare seismic responses in the frequency range around the acoustic cut-off frequency.

\section{Discussion}

The initial results from the Kepler mission have demonstrated its tremendous capability for studying stellar activity and oscillations. In particular, the previous observing programs have discovered that flares, during which stellar brightness dramatically increases for short periods of time, are common in both cool and hot stars. Previously, stellar flares were associated with active M-type stars, the UV Ceti variables, thought to be similar to solar flares, which represent a sudden release of magnetic energy accumulated in the coronal part of sunspot regions in the form of high-energy particles, which heat the lower atmosphere. However, the white-light emission in solar flares is rare, and there are only few cases when it has been unambiguously observed in the intergrated Sun-as-a-star observations. The stellar flares can be four orders-of-magnitude more powerful. This might be due to bigger sunspot regions generated by a more efficient dynamo process, because many of the flaring stars rotate faster than the Sun. However, there is an alternative point of view that the flares may be due to interactions with close companions, 'hot Jupiters'. The discovery of similar flares on hot A-type stars with a very shallow outer convection zone and without strong magnetic fields (Balona 2012) raises additional problems with the dynamo origin of the flare energy.

\section{References}

Balona, L. A. 2012, MNRAS, 423, 3420

Bryson, S., Kosovichev, A., \& Levy, D. 2005, Physica D Nonlinear Phenomena, 201, 1

Chaplin, W. J., Basu, S., Huber, D., et al. 2014, ApJS, 210, 1

Contadakis, M. E. 2012, in: I. Papadakis \& A. Anastasiadis (eds.), 10th Hellenic Astronomical Conference, p. 27

Contadakis, M. E., Avgoloupis, S. J., \& Seiradakis, J. H. 2012, AN, 333, 583

Jakimiec, J. \& Tomczak, M. 2012, Solar Phys., 278, 393

Karoff, C. \& Kjeldsen, H. 2008, ApJ, 678, L73

Koljonen, K. I. I., Hannikainen, D. C., \& McCollough, M. L. 2011, MNRAS, 416, L84

Kosovichev, A. G. 2006, Solar Phys., 238, 1

Kosovichev, A. G. 2009, AIP-CS, 1170, 547

Maehara, H., Shibayama, T., Notsu, S., et al. 2012, Nature, 485, 478

Qian, S.-B., Zhang, J., Zhu, L.-Y., et al. 2012, MNRAS, 423, 3646

Walkowicz, L. M., Basri, G., Batalha, N., et al. 2011, AJ, 141, 50 